\title{
CII. Manometric observations at the poles of the electric arc
}

\section{H.E.G. Beer M.Sc. \& Professor A.M. Tyndall}

To cite this article: H.E.G. Beer M.Sc. \& Professor A.M. Tyndall (1921) CII. Manometric observations at the poles of the electric arc , Philosophical Magazine Series 6, 42:252, 956-971, DOI: $10.1080 / 14786442108633836$

To link to this article: http://dx.doi.org/10.1080/14786442108633836

Published online: 08 Apr 2009.

Submit your article to this journal $₫$

Џ Article views: 2

Q View related articles $\square$

Citing articles: 3 View citing articles 5 
CII. Manometric Observations at the Poles of the Electric Are. By H. E. G. BeEr, M. Sc. (Bristol), and Professor A. M. TYNDALL *.

THE recent work of Duffield, Burnham, and Davis (Phil. Trans. A. vol. 220. p. 109, 1919 ; Proc. Roy. Soc. A. vol. xevii. p. 326,1920$)$ on the pressure on the poles of the electric. arc has revived interest in the problem of the mechanism of the transmission of current in the arc. In their first paper, when discussing the possible causes of the effect which they obtained, they drew attention to some early experiments of a different character by Dewar in 1888 . Dewar made observations on the hydrostatic pressure developed at carbon electrodes drilled centrally along their length. He found that a positive pressure of the order of 1 or $2 \mathrm{~mm}$. of water was developed at the anode, whereas that at the cathode was either absent or slightly negative. As, however, further quantitative data were not given, it was felt that a full investigation of the effect might be profitable and possibly give some information on the relative importance of positive and negative ions as carriers of current in the arc. The following is an account of experiments that we have carried out on the problem.

From the start we suspected that the effect was an electric wind phenomenon and that its small magnitude was evidence of the fact that both signs of ion take part in the discharge, in which case the wind-pressure would be the difference between the effects of each ionic stream taken separately. The results have substantiated this view.

Now if throughout the arc the ionic flow is viscous and the current carried by each sign is strictly proportional to its mobility, then there will be no space-charge in the arc and consequently no wind-pressure. If, however, in any region an excess of one sign of ion is present, a wind will be set up by the motion of these ions to the appropriate electrode. The experimental study of the relation between wind-pressure, arc-length, and current has enabled us to show that the wind-producing regions are contined to the vicinity of the electrodes, and that therefore the main body of the arc has no net space-charge. It is therefore presumably a region in which intense ionization and recombination is going on.

Moreover, the wind-pressure method enables a very sensitive test to be made of the perfection of the balance

* Communicated by the Authors. 
between the two ionic streams. This may be seen by considering the magnitudes of the effects produced by each sign separately. Taking Chattock's expression for electric windpressure, a current density of 10 amperes in an arc of $1 \mathrm{~cm}$. length carried entirely by electrons of, let us say, mobility $10,000 \mathrm{~cm} . / \mathrm{sec} . / \mathrm{volt} / \mathrm{cm}$. would give rise to a difference of pressure between the electrodes of about $10^{5}$ dynes per sq. cm., i.e. about $100 \mathrm{~cm}$. of water. The corresponding calculation for the same current carried entirely by the relatively slow-moving positive ions gives a pressure difference of several atmospheres.

Now the results show that in the main body of the arc the gradient of pressure, if any, is less than $1 \mathrm{dyne}$ per $\mathrm{cm}$. of arc. The mobilities of the ions in an are are not known. But if it is àssumed that the negative ions are electrons, the ratio of the mobilities is at least 500. Accepting this value for the time being, this means that in this region only $1 / 5$ per cont. of the current is carried by positive ions*. Thus, on this view, though the presence of this positive ionic stream is essential to bring about the observed balance, the main outcome of this result is that for most practical purposes we may regard the whole of the current as being carried by electrons.

It should be pointed out, however, that the above numbers must be modified if any appreciable part of the current is carried (1) by negative ions of molecular magnitude (see the following paper by one of us, A. M T.), or (2) by ions not moving viscously or, to give the specific case postulated by Daffield, Burnbam, and Davies, by electrons projected from the cathode and travelling with a speed large compared with that which they would acquire in the field itself. In the latter case the estimated fraction of current carried by positives would be still less. But this matter is referred to again below.

\section{Experiments.}

The first experiments were made with a horizontal straight arc of soft carbons $1 \cdot 3 \mathrm{~cm}$. diameter, through each of which a hole $3.5 \mathrm{~mm}$. diameter had been bored by removing the soft core. All experiments were carried out at atmospheric pressure. The pressure at either anode or cathode was

* These experiments are briefly mentioned in a note in the Phil. Mag. (Dec. 1920). Since that time it has been shown that the residual windpressure therein referred to originates near the electrode and not throughout the body of the arc. 
observed by connecting it to one limb of a Chattock Fry tilting bubble gauge of the usual type. In general, the other limb was open to air-that is to say, to some point in or near the body of the arc at which the pressure was that of the surrounding atmosphere. The particular instrument used responded to a difference of pressure of about $1 / 1000 \mathrm{~mm}$. of water.

An image of the arc was focussed on a screen so that the distance between the carbons could be maintained at required values and simultaneous readings taken of current and manometer pressure for various lengths of arc. At intervals the carbons were adjusted by filing to a standard shape. The Dewar effect was readily olitained-namely, an excess pressure at the anode for a silent arc and a comparatively négligible pressure which was sometimes negative at the cathode; also there was a greatly reduced value of anode pressure when the are was hissing.

But the effects were variable and difficult to correlate with one another, even when all readings for which the luminous patch on the electrode was not centred round the hole, were discarded. An observation that the anode pressure seemed to decrease with time suggested either that the Dewar effect was not an electrical wind-pressure phenomenon at all or that there was some superposed disturbing effect.

It is well known that earbon contains occluded gases. If the end of the carbon is raised to incandescence these gases will be gradually evolved, and if any of this evolution occurs from the walls of the hole leading to the manometer an excess pressure will be developed in the hole and at the gauge, the value of this pressure being dependent upon the rate of evolution of gas and the amount of constriction between the heated region of the hole and the outside air.

This view was easily put to test by using carbons bored as before, except that the last centimetre of carbon at the arc end was pierced with a hole $1.0 \mathrm{~mm}$. diameter instead of $3.5 \mathrm{~mm}$. If the effect was due to the evolution of gas, a much greater resultant pressure at the gauge was to be expected, because the constriction between the inner limit of the heated region and the outside air is approximately inversely proportional to the 4 th power of the radius of the bole and the heated surface area of the inner wall to the first power.

This was found to be the case, pressures as much as a hundredfold greater being observed in certain circumstances. Since we should expect that in time the end of the carbon would be denuded of its absorbed gas by incandescence, the 
variation of pressure with time from the moment of starting the are was studied. The results are shown in curves (1 A, B, C, D) for cathorle, (2 A, B, C, D) for mode. Curves $1 \mathrm{~A}$ and $2 \mathrm{~A}$ definitely show th t evolution of gas

Curves I. and II.

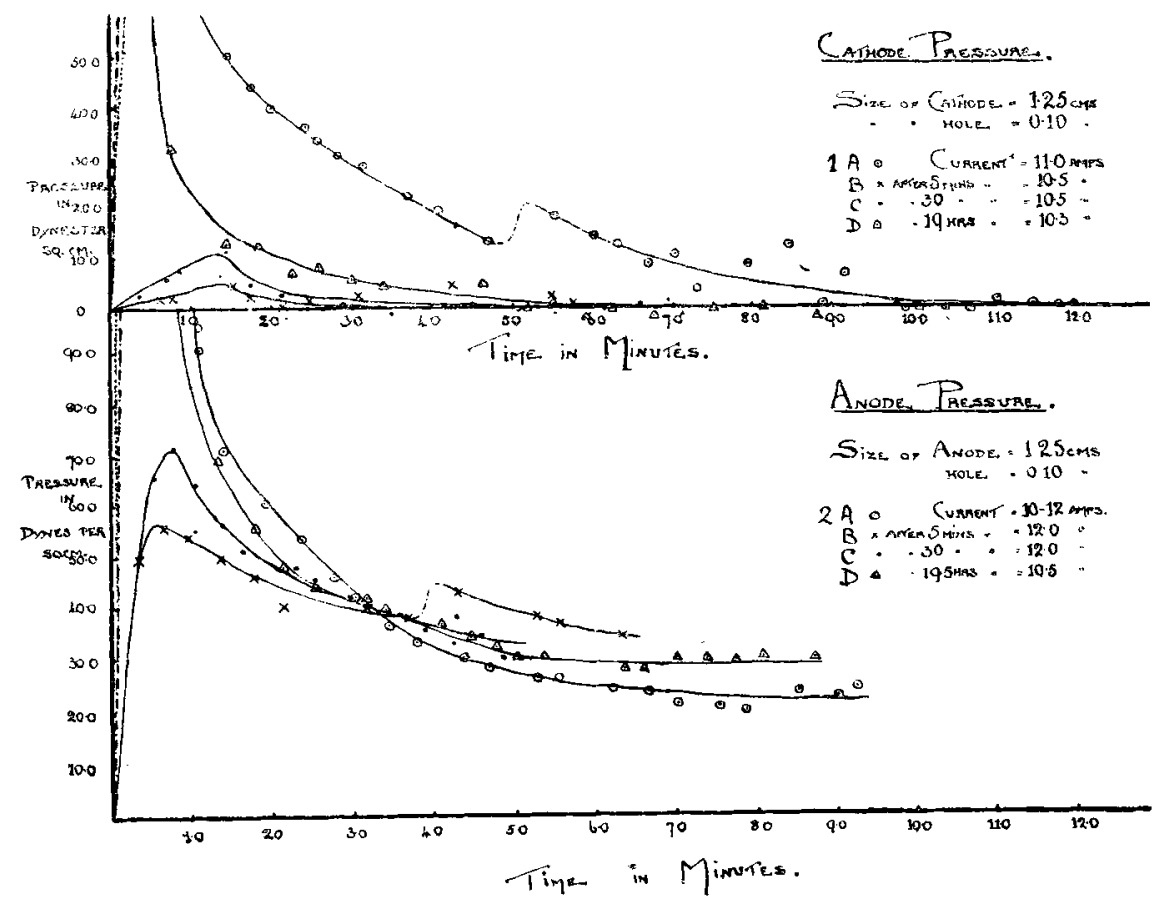

is at first the main cause of the pressure. The rate of evolution rises as the carbon warms and falls afterwards as the supply of absorbed gas is exhausted. Curves B, C, and $D$ are observations taken with the same carbons untouched. There was an interval of 5 minutes between the end of $A$ and the beginning of $B$, of 30 minutes between $C$ and $B$, and a whole night between $C$ and $D$. It is clearly seen that while a little re-absorption of gas took place in the 5 minutes' interval, it is mire obvious after a 30 minutes' interval. From the form of curves $1 \mathrm{D}$ and $2 \mathrm{D}$ the recovery of gas, except in the deeper-seated part of the carbon, would appear to be practically completed over night. 
Experimen's with Gas-denuded Carbons.

It appeared, however, that after the carbon had been denuded of its gases there was still a residual pressure which might possibly be attributed to an electric wind. This was borne out in the following long series of experiments with carbons which had been as far as possible denuded of gas by running the arc at a high current for 5 minutes before readings were taken, the current used for denudation being in all cases greater than that of which pressure measurements were subsequently taken.

The carbons used were filed down to a diameter of about $8 \mathrm{~mm}$., partly to ensure a more complete denudation of gas and still more to minimize as far as possible the tendency of the arc to leave its central position. This tendency was one of the main experimental difficulties of the work Considerable care and patience had to be exercised before a satisfactory set of readings could be obtained, because lack of centrality was often a matter of degree only. If the discharge shifted - as it frequently did-to one side of the carbon, this was immediately apparent without looking at the arc, as the gauge readings dropped instantly. But when it was nearly central, it was quite possible to mistake the appreciable reading which was obtained for the maximum reading which would have resulted from perfect centrality. In all cases of doubt the arc was adjusted and kept burning for some time, and the miximum gauge reading during this period was the one recorded.

For small currents and large holes, however, the incandescent patch of light on the anode was smaller than the hole itself, and it is doubtful how far the readings then are quantitatively valuable except for purposes of comparison. For the cathode with any hole buta small one this was always the case, and the readings were most variable and uncertain.

Another experimental difficulty was "hissing," which considerably restricted the scope of the observational work. It was particularly noticeable with post-war carbons. When the are was hissing, the luminous patch on the anode was quivering and travelling about, with the result that the effective pressure was greatly reduced on that electrode owing to the departure from centrality, if from no other cause as well. On the other hand, hissing caused the cathode pressure, which was generally negative for a large hole, to become positive.

It might also be mentioned that to obtain consistent readings for such minute pressures, precautions had to be 
taken to eliminate disturbances due to heating effects in the tubes Jeading to the gauge. These had to be kept strictly horizontal, close together, and screened with the gauge from the arc by asbestos sheets.

For the observation and measurement of some of the residual pressures at low currents, the gauge referred to above was not sensitive enough, and a Fry diaphragm gauge (Phil. Mag. vol. xxv. 6th ser. p. 494) was substituted for it. 'This was used in two grades of sensitivity : in one case the sensitivity was $\cdot 0076$ dyne per $\mathrm{mm}$. scale deflexion, and in the other 0205 dyne per $\mathrm{mm}$. It may be noted that in no case, except with the new undenuded carbons, were pressures recorded so high as the value 1 to $2 \mathrm{~mm}$. of water which Dewar quotes for an anode in his paper. Nevertheless, for the size of the hole used the signs of the effects are in agreement with those of Dewar-namely, a positive residual pressure at the anode and a pressure which was usually negative at the cathode.

Before considering theoretical explanations of these effects, two tests were applied :-

(a) A reading was taken with a denuded carbon. The current was then greatly increased for a few minutes, after which it was reduced again to its previous value and the reading immediately repeated. The absence of any change was taken to indicate that the reading was uninfluenced by the evolution of gas. There was no evidence of such evolution in any of the results given below, though naturally those taken with carbons of wide bore are less susceptible to this effect. But it should be mentioned that, particularly in the case of the anode, a fresh surface at the end of the hole is continually being exposed by the eating away of the electrode, and if this effect extends up the hole for even a short distance the evolution of gas may never be negligible. Whatever may be said, therefore, as to the true magnitude of the residual positive pressures-and this is discussed further below-there is every reason to suppose that the negative pressure often observed at the cathode is a true effect uninfluenced by evolution of occluded gases (see also below). It may be noted that the above remarks do not apply to the evolution of carbon vapour in the hole; this can produce no permanent pressure on the gauge.

(b) The possible influence of convection currents in the arc was studied. It was found that for a given current and length of arc the pressure reading was not affected by placing a plate of fused silica just over or just under the arc, though 
962 Mr. Beer and Prof. Tyndall on Manometric

a profound modification of the convection current must have been brought about by this means. Again, the carbons were enclosed in a horizontal quartz tube $1 \frac{1}{4} \mathrm{in}$. in diameter and closed at one end. Readings under these conditions were very difficult to obtain, and it was almost impossible to maintain a silent ceutral burning arc. But, so far as they went, they gave no evidence of modification as a result of the prevention of convection by the tube.

These results are consistent with the view that the pressures have their origin in the are itself, and arise from ionic streams giving rise to a residual wind-pressure. Evidence as to the particular region or regions in the arc in which this wind is generated can be deduced from a study of the variation of pressure with length of are at constant current. For this purpose the following observations were made. It is convenient to deal with the anode and cathode results separately.

Anode pressure.

Values of pressure and length of arc for various currents with holes of different sizes were obtained. For reasons which will be clear later, only those for a hole of diameter $1.5 \mathrm{~mm}$. are inserted in this account, and these are shown graphically in curves III.

It will be seen that fluctuations of considerable magnitude occur ; these could be traced to slight departures from true centreing on the hole of the luminous patch on the electrode. In fact, the readings are so sensitive to this that much work was necessary before we were assured that results such as are given there represent the facts.

With each observation great care was taken that the luminous patch was centred as perfectly as possible. This was easy with short arcs, but with long ones burnt in the ordinary way there was a marked lack of centrality-due no doubt to the action of convection currents on the flame, as the arc was horizontal; but by holding a quartz plate horizontally just over the arc, it was found that the patch could be brought back on to the hole, and "central" readings for all lengths of arc were thus obtained.

Curves III. show that the pressure at the anode is practically, if not wholly, independent of the length of the arc, the apparent slope of two of the lines being not more than might be attributed to experimental errors.

This result strongly suggests that the pressure originates in the region quite close to the anole itself, and experiments 
Observations at the Poles of the Electric Arc.

with different sizes of hole and current afford additional support for this view. Readings were obtained for holes

Curres III.

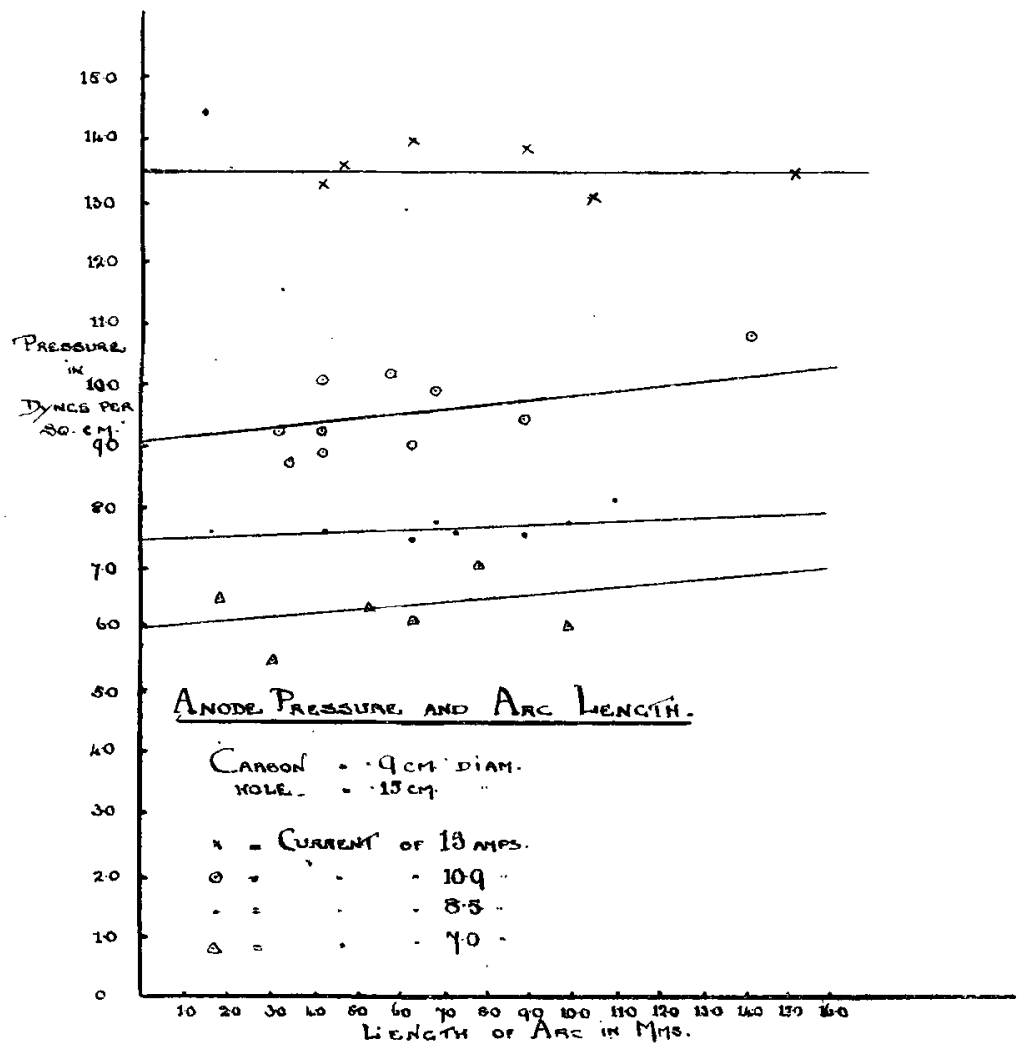

up to $5 \mathrm{~mm}$. in diameter, and it was found that the pressure decreased as the hole was male larger. Now there are three possible causes for this effect, namely :-

(1) If the pressure is set up by evolved gas, its effect will be reduced by the reduction in constriction.

(2) If the pressure reaches a maximum at the centre of the incandescent patch and falls off towards its circumference, the average pressure over a large hole will be less than over a small one when properly centred.

(3) If the origin of the pressure lies in a layer quite close to the electrode, as the pressure-arc length curves suggest, 
the true pressure at the surface of the electrode will only be recorded by using a hole which is small compared with the thickness of this layer.

The evidence given above is definitely against (1). Our experiments furnish no evidence for or against (2), whereas it can be shown that they give strong quantitative support for (3).

The experimental troubles brought about by the constriction due to a fine hole place a lower limit on the diameter which can be successfully used, and, except in a few cases (when the pressure rose still higher), no hole of diameter less than 1.7 mm. has been experimented with--a length which is almost certainly enormously greater than the thickness of the pressure layer.

Given, however, that the pressure-producing layer is very thin and that the current density at the luminons patch is independent of the size of the hole, it is easy to see that the pressure observed at a relatively large hole at the centre of the patch is approximately proportional to the circumference of the hole at which the pressure is communicated and inversely proportional to the area of the hole. In other words, we might expect on hypothesis (3) that the observed pressure will be approximately inversely proportional to the diameter of the hole. When the hole is comparable in size with the luminous patch, it is impossible to obtain true centrality of hole, but it is reasonable to extend the argument to the case of a displaced hole, provided the conditions are similar for all the holes used. An analysis of the following results for different currents and sizes of hole substantially verify the hypothesis.

In every case, whatever the size of the hole, an increase of current is accompanied by an increase of pressure at the anode. Typical results are shown in curves IV. The values of size of hole and length of arc applying to curves A, B, and $\mathrm{C}$ are given on the graph.

Graph $\mathrm{D}$ is the only line passing through the origin. The readings for this were taken under conditions in which the greatest care was observed in ensuring centrality of the arc, the hole being always smaller than the luminous patch so that this was possible. In curves $A, B$, and $C$, on the other hand (except porhaps at the largest currents), the hole was always larger than the luminous patch and the centreing consequently not perfect.

The conditions of centreing were, however, sufficiently similar to enable us to use the three curves, A, B, and (: (as well as others which for sake of clearness are not 
Observations at the Poles of the Electric Arc. $\quad 965$

included in the graph), to test the above relationship. The results are shown in Table I. for three values of current,

Curves IV.

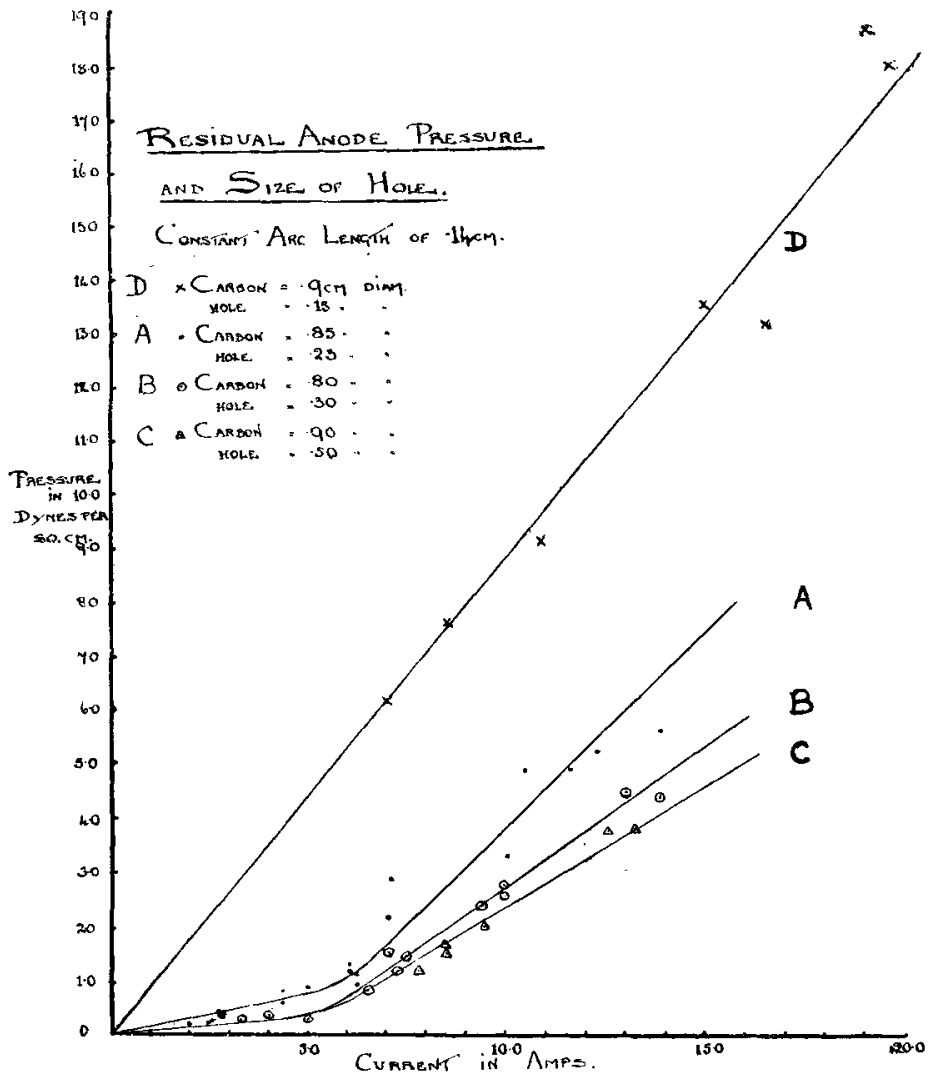

the test of the hypothesis lying in the values of the product of the diameter of hole and the pressure observed, given in each vertical column :-

\section{Table I.}

Diameter $\times$ manometer reading.

Diameter of hole in $\mathrm{mm}$.

$1 \cdot 7$

$2 \cdot 3$

$3 \cdot 0$

$4 \cdot 0$

50

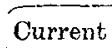

$7 \cdot 5$ amps.

23

28

25

32

35
Current

$10.5 \mathrm{amps}$.

52

53

48

55

59
Current

14.5 amps.

85

82

72

89

96 
If we omit the results for the 5-mm. hole, it will be seen that the values of the product in each vertical column are reasonably constant considering the difficulties of obtaining reliable results for comparison. The increase in the value of the product for the larger holes is also not unexpected, because such a hole reduces appreciably the effective area of the electrode and causes an increase in the current density which is not allowed for. In our opinion, therefore, there is strong qualitative and quantitative evidence for the view that the Dewar pressure at the anode is due to a negative space-charge in a layer of gas very close to the electrode itself.

With regard to graph D, some of the points on it are taken from those in curves III., selecting a value of arclength of $1.4 \mathrm{~mm}$; the other points included are from similar pressure arc-length results not plotted in curves III.

It' seems quite clear, then, that for a truly central are, as given by curve $D$, the current and anode pressure are proportional. Under other conditions the readings are too low, due to the fact that the true average pressure at the luminous patch is not felt at the hole. At low currents and large holes it was found, indeed, that for a non-central arc the pressure was in some cases actually reversed. This effect, which in all cases was very minute, is referred to again below.

\section{Cathode pressure.}

Quantitative work at the cathode is almost impossible, owing to the fact that the luminous patch is much smaller and will rarely remain centred on a small hole in the electrode long enough to obtain readings which are definite except in sign. It is simple to obtain readings of a sort with ares which are not quite central on the cathode; and in order that they may be correlated with similar curves for the anode they are given in curves $\mathrm{V}$.

For large currents in all these non-central cases the pressures were negative. In the case of the large holes the luminous pateh was sometimes seen to be actuaily playing right inside the loole at a point on its wall. In the case of the small holes the spot was about half on and half off the hole. With small currents the pressures were often but not always reversed in sign, but were so irregular that it is impossible to include them in any graph. 
Observations at the Poles of the Electric Arc. $\quad 967$

Finally, careful readings were taken with a hole of $1.5 \mathrm{~mm}$. diameter, selecting only the rare occasions on which the arc was really central. In every such case a

Curves V.

Tesidual Cariode Pressure anosize of Hour.

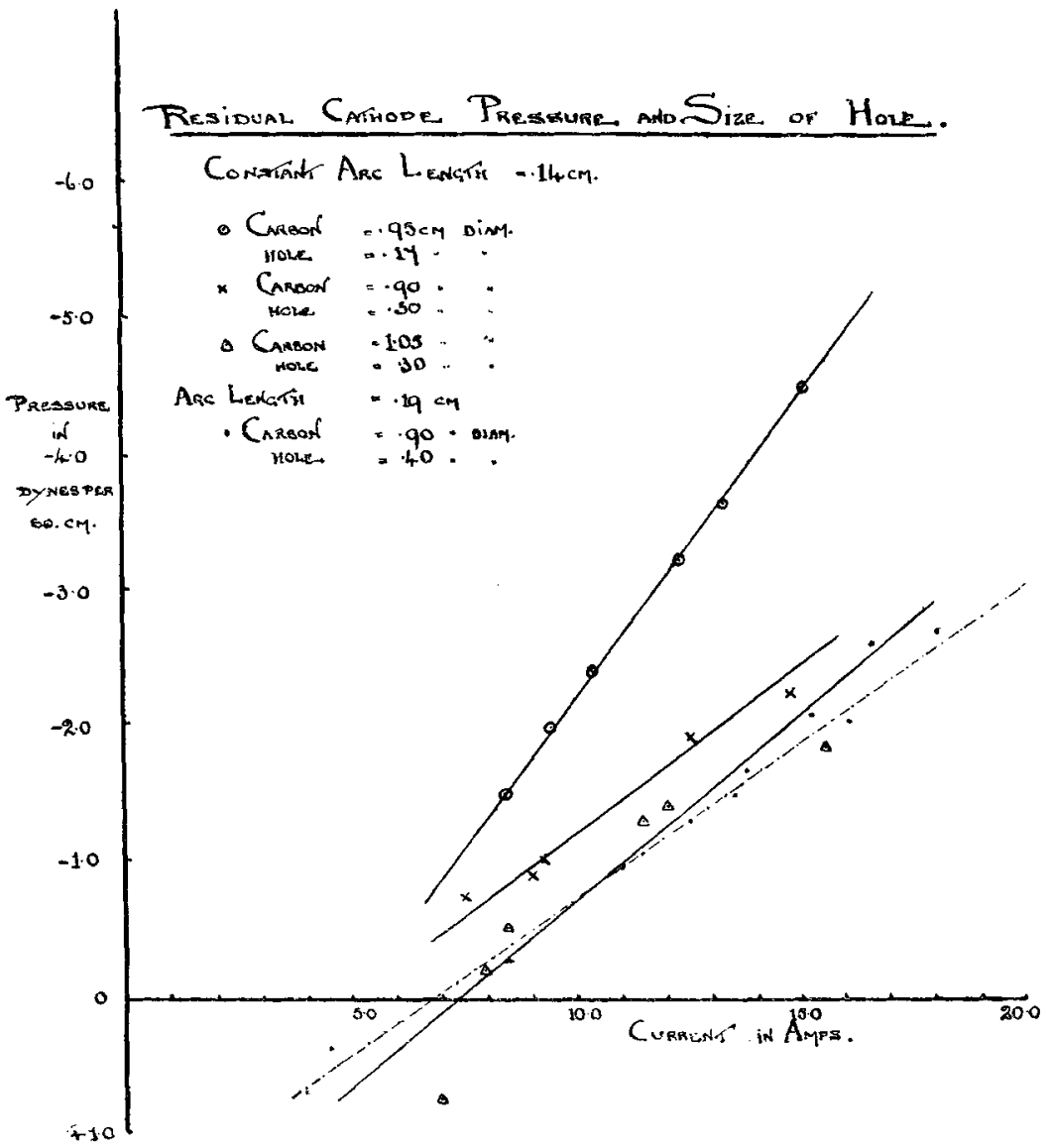

relatively large positive pressure was recorded. This result was confimed by a few readings taken with a smaller hole $1.1 \mathrm{~mm}$. in diameter. The order of magnitude of the effect is given by the results shown in Table II, though the spot rarely remained central long enough to ensure that the gauge had filled up to the full pressure at the mouth of the hole :- 
Table II.

\begin{tabular}{|c|c|c|c|c|c|}
\hline \multicolumn{3}{|c|}{$\begin{array}{l}\text { Oathode }=0.9 \mathrm{~cm} . \text { diam. } \\
\text { Hole }=0.15 \mathrm{~cm} .\end{array}$} & \multicolumn{3}{|c|}{ Catbode $=0.9 \mathrm{~cm}$. diam. } \\
\hline $\begin{array}{l}\text { Arc-length } \\
\text { in mm. }\end{array}$ & $\begin{array}{l}\text { Current in } \\
\text { amperes. }\end{array}$ & $\begin{array}{l}\text { Pressure } \\
\text { in dynes } \\
\text { per sq. cm. }\end{array}$ & $\begin{array}{l}\text { Arc-length } \\
\text { in } \mathrm{mm} \text {. }\end{array}$ & $\begin{array}{l}\text { Current in } \\
\text { amperes. }\end{array}$ & $\begin{array}{l}\text { Pressura } \\
\text { in dynes } \\
\text { per sq. cm. }\end{array}$ \\
\hline- & 13.5 & $6 \cdot 9$ & 70 & 135 & $24 \cdot 7$ \\
\hline & & & $7 \cdot 0$ & $17 \cdot 0$ & 132 \\
\hline $1: 3$ & $17 \cdot 0$ & $18 \cdot 6$ & 50 & $18 \cdot 1$ & $5 \cdot 7$ \\
\hline & & & 60 & $19 \cdot 5$ & 31.8 \\
\hline
\end{tabular}

For this reason it was not found possible to obtain any definite relationship between arc-length and pressure or between current and pressure, and so to rule out evolution of gas as the cause of the pressures, as was possible for the anode. But the experiments at least afford no evidence of regular variation of pressure with arc-length; and as the temporature is lower and the current more concentrated at the cathode than at the anode, there is now still more reason than in the case of the anode to suppose that we are dealing with the effects of space-charge near the electrode and not with those of gas evolution.

It is clear that with the large holes used by Dewar he did not observe the positive cathode pressure of a perfectly central arc, but was dealing with negative pressures similar to those shown in curves V. Comparison of these with the anode results under similar conditions show that in agreement with him the cathode negative pressures are smaller than the anode positive pressures, though the difference in magnitude is perhaps not so great as his remarks suggest. $\mathrm{He}_{\mathrm{e}}$ did not state, however, what range of current he was using, and the fact that the luminous patch takes up a more or less central position on the anode more readily than on the cathode may well have led to an under estimate by him of the cathode pressure.

\section{Anode and Cathode together.}

Lastly, some attempts were made to measure the total difference of pressure between the electrodes by connecting the two carbons to opposite sides of the gauge at the same time. The purpose of these measurements was partly to check the previous results, but mainly to obtain readings from which the effects of convection were eliminated. But the difficulties of hissing and of obtaining centrality of arc 
at both carbons at the same time made quantitative study quite impossible. The readings, such as they were, were consistent with the results obtained separately on each pole, and, taken in conjunction with the experiments on convection referred to above, support the view that this plays no fundamental part in the phenomena.

'The fact that there is no excess of ions of one sign in the body of the are was confirmed during the progress of the above investigations by some experiments which were carried out to detect the lateral loss of momentum, which will, in general, take place when an unbalanced ionic stream crosses the arc. With short ares and flat poles of relatively large area this can, in any case, certainly be regarded as very small in amount. But its presence might be detected if it could be accentuated by increasing the arc-length and decreasing the pole diameter. Accordingly, the are was struck inside a quartz tube of $1 \frac{1}{2}$ inches diameter, the carbons lying along the axis of the tube. The anode was solid, and was filed down to a diameter of $0.7 \mathrm{~cm}$. and was made pointed. The anode end of the quartz tube was closed except for an opening to the pressure-gauge; the catbode end was open to the air.

Under these circumstances with the longer arcs that are possible in such a narrow tube, if there were an electric wind in the are from cathode to anode, a large fraction of its momentum would be transmitted laterally to the air in the quartz tube. This should lead to an excess pressure around the carbon at the anode end of the tube. Assuming all the momentum to be so transmitted, this pressure would be the Dewar pressure, reduced, however, in the ratio of the current-receiving area of the anode to the area of the quartz tube. This ratio was of the order of 0.03 for 20 amperes.

Definite results were diffioult to obtain, partly owing to hissing and partly to the great generation of heat. In some cases with a current of about 20 amperes a deflexion of from 2 to $4 \mathrm{~mm}$. of scale was obtained with the diaphragm gauge in its most sensitive form-i. e., 0076 dyne per mm. Between large electrodes giving no sideway loss this is equivalent to a Dewar pressure of not more than 1.1 dynes cm. ${ }^{2}$ for 20 amperes. But as it was independent of the direction of the current, it is reasonable to attribute it not to wind, but to a slight change in density of the air in the tube leading to the gauge, owing to its proximity to the arc and the hot quartz tube.

This therefore indirectly confirms the view that the Dewar pressure does not originate from wind-producing ions moving in the main body of the are.

Phil. Mag. S. 6. Vol. 42. No. 252. 1ee. 1921. $3 \mathrm{~S}$ 


\section{Discussion of Results.}

The above experiments show most clearly that the body of the arc possesses no net chirge, and that this statement holds to within a very short distance from the anode and probably-though on this point the evidence is not so definite-from the cathode as well.

Compared with any slope of potential method of investigating space-charge distribution in the arc, the windpressure method is extraordinarily sensitive. In the body of the arc, therefore, the balance between the two ionic streams is perfectly maintained.

If the negative ions are electrons, the ratio of their mobility to that of the positive ions is presumably at least 500 ; so that, except close to the electrodes, the current carried by positive ions cannot be more than one-fifth per cent. of the total. If, however, any of the negative ions are of atomic magnitude, this percentage is too low.

The view that the arc is in a highly dissociated state is therefore confirmed. Production and recombination of ions is continually taking place, and the ions in drifting to the electrodes under the influence of the fieid give rise to spacecharges near the electrodes. It has been shown above that the pressure observed at the anode is in quantitative agreement with the view that it is produced by the motion of a space-charge of negative ions in a thin layer close to the electrode; but the data are not sufficient to give the magnitude of the charge or the thickness of the layer in which it is appreciable. We may, however, make a rough guess if we take a current of 10 amperes and assume the anode pressure at an ininitesimal hole to be, let us say, 50 dynes/sq. cm. (or five times that observed at the smallest hole used). If we further assume the current to be carried entirely by negative ions of mobility $10,000 \mathrm{~cm} . / \mathrm{sec} . / \mathrm{volt} / \mathrm{cm}$., it may be shown from the wind-pressure formula that the thickness is $10^{-3} \mathrm{~cm}$. The thickness would be far less if the negative ions in the arc are of molecular and not electronic magnitude. On the other hand, the presence of positive ions taking part in the flow of the current would increase the estimated thickness.

That some positive ions are present at the anode surface is almost certain on thermionic grounds. Also they afford a simple explanation of the small reversed readings, referred to above and obtained in some cases with a displaced arc. The region outside the luminous area where the main current arises is red-hot, and is therefore presumably emitting positive ions. If these form an unbalanced stream of positive 
ions travelling right across the arc and carrying a current of less than a microampere, a reversed pressure of the required magnitude wonld be set up. If, as is more likely, they travel a shorter distance and then become merged in the body of the arc and balanced there by negatives, the current they must carry would be larger, but still small compared with the total current in the are.

At the cathode the pressure effects appear to be rounhly similar to those at the anode, though much more erratic. When the luminous patch really covers the hole, the pressure is positive and may be quite large; but if the pateh is off the centre-either playing on the wall of the hole in the case of a small patch, or partly on and partly off in the case of a large one,- the pressure becomes negative.

This suggests that, as at the anode, there is a space-charge (in this case positive) at the luminous spot itself; while from the hot carbon around it, negative thermions are emitted which travel for an appreciable distance before ceasing to produce suction by their drag on the gas.

This drag, if it exists, is not to be confounded with the drag which would result from the slowing-down of electrons emitted at high speeds from the cathode, as postulated by Duffield. The latter, even if it could appreciably affect the manometer, would not give a suction anything like large enough. The drag here referred to is the steady pull of the field upon unbalanced ions, and may be quite large.

But speculation on this point is the less necessary, as it has no effect on the main conclusions of the work.

\section{Summary.}

(1) By a wind-pressure method it has been shown to a degree of accuracy unobtainable by polential slope methods that the body of the are at atmospheric pressure contains no net space-charge, and that therefore the proportion of the currents carried by the two signs of ion is that of their. respective mobilities.

(2) An excess hydrostatic pressure is set up at the anode by the motion of an unbalanced space-charge of negative ions in a very thin layer close to the anode. The results indicate that this pressure is proportional to the current carried by the arc.

(3) The distribution of pressure in the neighbourhood of the cathode is much more complicated, being sometimes positive and sometimes negative. The conditions under which given results are obtained are discussed, and a tentative explanation in terms of space-charge suggested. 